\title{
Fourth order 4-stages improved Runge-Kutta method with minimized error norm
}

\begin{abstract}
In this paper the improved Runge-Kutta method of order four with 4-stages for solving first order ordinary differential equation is proposed. The method is based on classical RungeKutta (RK) method also can be considered as special class of two-step method. Here, the coefficients of the method are obtained using the minimization of the error norm up to order five. The improved method with only 4-stages is more accurate than fourth order 4-stages RK method. Therefore it is computationally more efficient than the existing RK method. A number of test problems are solved and the numerical results compared with the existing RK method are given.
\end{abstract}

Keyword: Improved Runge-Kutta method; Minimization of error norm; Ordinary differential equations; Two-step method 